\title{
Global existence and asymptotic behavior of classical solutions to Goursat problem for diagonalizable quasilinear hyperbolic system
}

\author{
Jianli Liu' and Kejia Pan ${ }^{2,3 *}$
}

\footnotetext{
* Correspondence: kjpan@yahoo.cn ${ }^{2}$ Key Laboratory of Metallogenic Prediction of Nonferrous Metals, Ministry of Education, School of Geosciences and Info-Physics, Central South University, Changsha 410083, China

Full list of author information is available at the end of the article
}

\begin{abstract}
In this article, we investigate the global existence and asymptotic behavior of classical solutions to Goursat problem for diagonalizable quasilinear hyperbolic system. Under the assumptions that system is strictly hyperbolic and linearly degenerate, we obtain the global existence and uniqueness of $C^{1}$ solutions with the bounded $L^{1} \cap L^{\infty}$ norm of the boundary data as well as their derivatives. Based on the existence result, we can prove that when $t$ tends to in nity, the solutions approach a combination of piece-wised $C^{1}$ traveling wave solutions. As the important example, we apply the results to the chaplygin gas system.

Mathematics Subject Classi cation (2000): 35B40; 35L50; 35Q72.
\end{abstract}

Keywords: Goursat problem, global classical solutions, linearly degenerate, asymptotic behavior, traveling wave solutions.

\section{Introduction and main results}

For the general first order quasilinear hyperbolic systems,

$$
\frac{\partial u}{\partial t}+A(u) \frac{\partial u}{\partial x}=0
$$

the global existence of classical solutions of Cauchy problem has been established for lin-early degenerate characteristics or weakly linearly degenerate characteristics with various smallness assumptions on the initial data by Bressan [1], Li [2], Li and Zhou [3,4], Li and Peng [5,6], and Zhou [7]. The asymptotic behavior has been obtained by Kong and Yang [8], Dai and Kong [9,10]. For linearly degenerate diagonalizable quasilinear hyperbolic systems with "large" initial data, asymptotic behavior of the global classical solutions has been ob-tained by Liu and Zhou [11]. For the initial-boundary value problem in the first quadrant Li and Wang [12] proved the global existence of classical solutions for weakly linearly degenerate positive eigenvalues with small and decay initial and boundary data. The asymptotic behavior of the global classical solutions is studied by Zhang [13]. The global existence and asymptotic behavior of classical solutions of the initial-boundary value problem of diagonal-izable quasilinear hyperbolic systems in the first quadrat was obtained in [14].

However, relatively little is known for the Goursat problem with characteristic boundaries. Global existence of the global classical solutions for the Goursat problem

(c) 2012 Liu and Pan; licensee Springer. This is an Open Access article distributed under the terms of the Creative Commons Attribution License (http://creativecommons.org/licenses/by/2.0), which permits unrestricted use, distribution, and reproduction in any medium, provided the original work is properly cited. 
of reducible quasilinear hyperbolic system was obtained in [15]. Under the assumptions of boundary data is small and decaying, the global existence and asymptotic behavior to classical solutions can be obtained by Liu $[16,17]$. The asymptotic behavior of classical solutions of Goursat problem for reducible quasilinear hyperbolic system was shown in [18].

In this article, we consider the following diagonalizable quasilinear hyperbolic system:

$$
\frac{\partial u_{i}}{\partial t}+\lambda_{i}(u) \frac{\partial u_{i}}{\partial x}=0
$$

where $u=\left(u_{1}, \ldots, u_{n}\right)^{T}$ is unknown vector-valued function of $(t, x) . \lambda_{i}(u)$ is given by $C^{2}$ vector-valued function of $u$ and is linearly degenerate, i.e.,

$$
\frac{\partial \lambda_{i}(u)}{\partial u_{i}} \equiv 0
$$

The system (1) is strictly hyperbolic, i.e.,

$$
\lambda_{1}(u)<\cdots<\lambda_{m}(u)<0<\lambda_{m+1}(u)<\cdots<\lambda_{n}(u)
$$

Suppose that there exists a positive constant $\delta$ such that

$$
\lambda_{i+1}(u)-\lambda_{i}(v) \geq \delta, \quad i=1, \ldots, n-1
$$

Consider the Goursat problem for the strictly quasilinear hyperbolic system (1), in which the solutions to system (1) is asked to satisfy the following characteristic boundary conditions:

$$
x=x_{1}(t): \quad u=\phi(t)
$$

and

$$
x=x_{n}(t): \quad u=\psi(t)
$$

where $x=x_{1}(t)$ and $x=x_{n}(t)$ are the leftmost and the rightmost characteristics passing through the origin $(t, x)=(0,0)$, respectively, such that

$$
\left\{\begin{array}{l}
\frac{d x_{1}(t)}{d t}=\lambda_{1}(\phi(t)) \\
x_{1}(0)=0
\end{array}\right.
$$

and

$$
\left\{\begin{array}{l}
\frac{d x_{n}(t)}{d t}=\lambda_{n}(\psi(t)) \\
x_{n}(0)=0
\end{array}\right.
$$

moreover,

$$
l_{1}(\phi(t)) \phi^{\prime}(t) \equiv 0
$$

and

$$
l_{n}(\psi(t)) \psi^{\prime}(t) \equiv 0
$$


where $\varphi(t)=\left(\varphi_{1}(t), \ldots, \varphi_{n}(t)\right)^{T}$ and $\psi(t)=\left(\psi_{1}(t), \ldots, \psi_{n}(t)\right)^{T}$ are any given $C^{1}$ vector functions satisfying the conditions of $C^{1}$ compatibility at the origin $(0,0)$ :

$$
\phi(0)=\psi(0)
$$

and

$$
\lambda_{n}(\phi(0)) \phi^{\prime}(0)-\lambda_{1}(\psi(0)) \psi^{\prime}(0)+A(\phi(0))\left(\psi^{\prime}(0)-\phi^{\prime}(0)\right)=0 .
$$

$l_{i}(u)$ be a left eigenvector corresponding to $\lambda_{i}(u)$ and $A(u)=\operatorname{diag}\left\{\lambda_{1}(\mathrm{u}), \ldots, \lambda_{n}(u)\right\}$.

Our goal in this article is to get the global existence and asymptotic behavior of the global classical solutions of the Goursat problem (1), (5), and (6) with "large" boundary data. With the assumptions that

$$
\begin{array}{ll}
\sup _{t \in R^{+}}\left\{\left|\phi^{\prime}(t)\right|+\left|\psi^{\prime}(t)\right|\right\} \doteq M<\infty, & \sup _{t \in R^{+}}\{|\phi(t)|+|\psi(t)|\} \doteq M_{0}<\infty \\
\int_{0}^{+\infty}\{|\phi(t)|+|\psi(t)|\} d t \doteq N_{1}<\infty, & \int_{0}^{+\infty}\left\{\left|\phi^{\prime}(t)\right|+\left|\psi^{\prime}(t)\right|\right\} d t \doteq N_{2}<\infty
\end{array}
$$

we can prove the following results:

Theorem 1.1. The above assumptions and the conditions of $C^{1}$ compatibility at the point $(0,0)$ are satisfied, the Goursat problem (1), (5), and (6) admits a unique global $C^{1}$ solutions $u=u(t, x)$ on the domain $D=\left\{(t, x) \mid t \geq 0, x_{1}(t) \leq x \leq x_{n}(t)\right\}$.

If the leftmost and rightmost characteristics are convex, we can get the following result:

Theorem 1.2. Under the assumptions of Theorem 1.1, there exists a unique piecewised $C^{1}$ vector-valued function $\Phi(x)=\left(\Phi_{1}(x), \ldots, \Phi_{n}(x)\right)^{T}$ such that

$$
u(t, x) \rightarrow \sum_{i=1}^{n} \Phi_{i}\left(x-\lambda_{i}(0) t\right) e_{i,} \quad t \rightarrow+\infty
$$

uniformly as $t$ tends to infinity, where $e_{i}=\left(0, \ldots, 0,1^{i}, 0, \ldots, 0\right)^{T}$.

Remark 1.1. If the system (1) is non-strictly hyperbolic but each characteristic has constant multiplicity, then the result is similar as Theorems above.

\section{Global existence of $\boldsymbol{C}^{\mathbf{1}}$ solutions}

In this section, we will obtain some uniform a priori estimate which also play an important role in the proof of Theorem 1.1. In order to proving the global existence of classical solutions of the Goursat problem (1), (5), and (6), we will prove that $\|u\|_{C^{1}(D)}$ is bounded. For any fixed $T \geq 0$, we denote $D_{T}=\left\{(t, x) \mid 0 \leq t \leq T, x_{1}(t) \leq x \leq x_{n}(t)\right\}$ and introduce

$$
\begin{aligned}
& w_{i}(t, x)=\frac{\partial u_{i}(t, x)}{\partial x}(i=1, \ldots, n), \quad W_{1}(T)=\sup _{0 \leq t \leq T} \int_{x_{1}(t)}^{x_{n}(t)}|w(t, x)| d x \\
& \tilde{W}_{1}(T)=\max _{i \neq j} \sup _{\tilde{C}_{j}} \int_{\tilde{C}_{j}}\left|w_{i}(t, x)\right| d t, \quad \tilde{U}_{1}(T)=\max _{i \neq j} \sup _{\tilde{C}_{j}} \int_{\tilde{C}_{j}}\left|u_{i}(t, x)\right| d t
\end{aligned}
$$




$$
\begin{aligned}
& \bar{W}_{1}(T)=\max _{i \neq j} \sup _{L_{j}} \int_{L_{j}}\left|w_{i}(t, x)\right| d t, \bar{U}_{1}(T)=\max _{i \neq j} \sup _{L_{j}} \int_{L_{j}}\left|u_{i}(t, x)\right| d t \\
& W_{\infty}(T)=\sup _{(t, x) \in D_{T}}\{\mid w(t, x)\}, \quad U_{\infty}(T)=\sup _{(t, x) \in D_{T}}\{\mid u(t, x)\}
\end{aligned}
$$

where $\tilde{C}_{j}$ stands for any given $j$ th characteristic $\frac{d x}{d t}=\lambda_{j}(u), L_{j}$ stands for any given radial that has the slope $\lambda_{j}(0)$ on the domain $D_{T}$.

Lemma 2.1. Under the assumptions of Theorem 1.1, there exists a positive constant $C$ such that, the following estimates hold

$$
\begin{aligned}
& \tilde{W}_{1}(T), \bar{W}_{1}(T), W_{1}(T) \leq C N_{2} \\
& \tilde{U}_{1}(T), \bar{U}_{1}(T) \leq C N_{1} e^{C N_{2}} \\
& W_{\infty}(T) \leq C M e^{C N_{2}} \\
& U_{\infty}(T) \leq C
\end{aligned}
$$

Remark 2.1. The positive constant $C$ is only depend on $\delta, M_{0}$ and independent of $M$, $N_{1}, N_{2}, T$. In the following, the meaning of $C$ is similar but may change from line to line.

Proof. For any fixed point $(t, x) \in D_{T}$, we draw the $i$ th characteristic $\tilde{C}_{i}: x=x_{i}(t)$ through this point and intersecting $x_{1}(t)$ or $x_{n}(t)$ at a point $\left(t_{*}, x_{1}\left(t_{*}\right)\right)$ or $\left(t_{*}, x_{n}\left(t_{*}\right)\right)$. Noting system $(1), u_{i}(t, x)$ is a constant along the $i$ th characteristic, then we have $u_{i}(t, x)=$ $\varphi_{i}\left(t_{*}\right)$ or $u_{i}(t, x)=\psi_{i}\left(t_{*}\right)$. Then

$$
\left|u_{i}(t, x)\right| \leq \sup _{t \in R^{+}}\left\{\left|\phi_{i}\left(t_{*}\right)\right|+\left|\psi\left(t_{*}\right)\right|\right\} \leq M_{0} \leq C
$$

Then, we obtain the estimate (23).

Differentiating (1) with respect to $x$ we can get

$$
\frac{\partial w_{i}}{\partial t}+\frac{\partial\left(\lambda_{i}(u) w_{i}\right)}{\partial x}=0
$$

We rewrite (25) as

$$
d\left(w_{i}(t, x)\left(d x-\lambda_{i}(u) d t\right)\right)=0
$$

Multiplying the system above by $\operatorname{sign}\left(w_{i}\right)$, we have

$$
d\left(\left|w_{i}(t, x)\right|\left(d x-\lambda_{i}(u) d t\right)\right)=0
$$

There are only the following cases(as shown in Figure 1):

Case 1 . For any fixed $t_{0} \in R^{+}$, let $\tilde{C}_{j}: x=x_{j}(t), j>1$ stands for any given $j$ th characteristic, passing through any point $A\left(t_{0}, x_{1}\left(t_{0}\right)\right)$ on the boundary $x=x_{1}(t)$ and intersects $t=T$ at point $P$. We draw an $i$ th characteristic $\tilde{C}_{i}: x=x_{i}(t)$ from $P$ downward, intersecting $x=x_{1}(t)$ at a point $B\left(t_{1}, x_{1}\left(t_{1}\right)\right)$. Without loss of generality, we assume $t_{0}<t_{1}$, then $j>i$. Integrating (26) in the region $A P B$ to get 

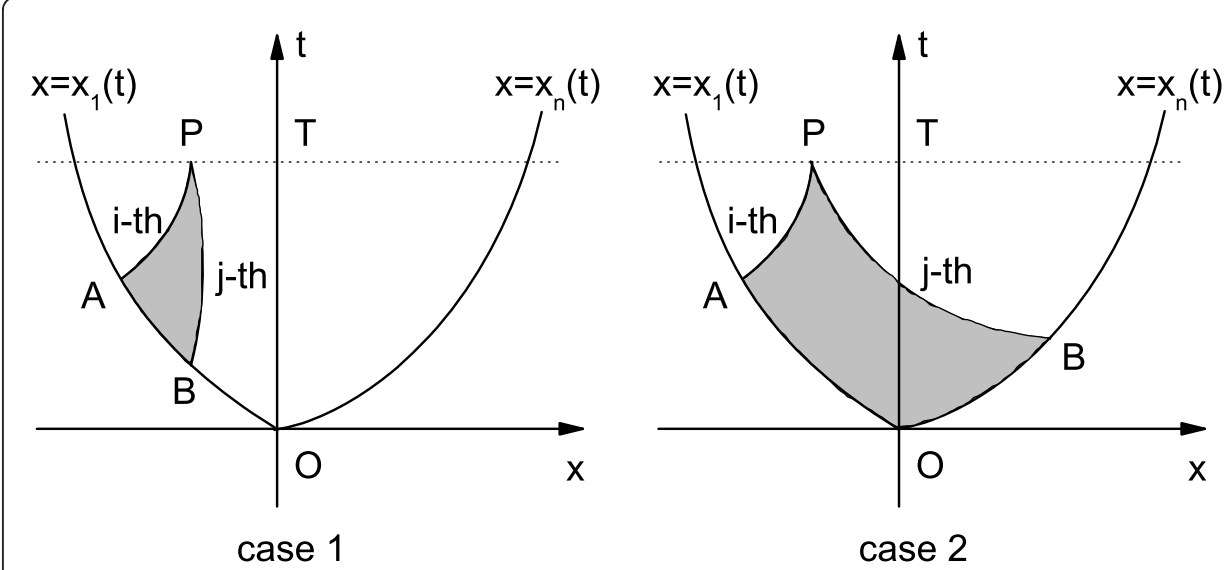

Figure 1 Cases in estimating $\tilde{W}_{1}(T)$

$$
\int_{\tilde{C}_{j}}\left(\lambda_{j}(u)-\lambda_{i}(u)\right)\left|w_{i}(t, x)\right| d t=\int_{t_{0}}^{t_{1}}\left(\lambda_{i}(u)-\lambda_{i}(u)\left|w_{i}\left(t, x_{1}(t)\right)\right| d t\right.
$$

Along the 1th characteristic, $\frac{d x}{d t}=\lambda_{1}(u)$, then $\frac{d t}{d x}=\frac{1}{\lambda_{1}(u)}$

$$
\frac{\partial u_{i}\left(t, x_{1}(t)\right)}{\partial x}=\frac{\partial u_{i}\left(t, x_{1}(t)\right)}{\partial t} \frac{\partial t}{\partial x}=\frac{\phi_{i}^{\prime}(t)}{\lambda_{1}(u)}
$$

Noting (4), (13), and (23), we can get

$$
\int_{\tilde{C}_{j}}\left|w_{i}(t, x)\right| d t \leq C \int_{0}^{\infty}\left|\phi_{i}^{\prime}(t)\right| d t \leq C N_{2}
$$

Case 2. For any fixed $t_{0} \in R^{+}$, passing through the point $A\left(t_{0}, x_{1}\left(t_{0}\right)\right)$, we draw $\tilde{C}_{j}: x=x_{j}(t)$ and intersecting $t=T$ at point $P$. We draw the $i$ th characteristic $\tilde{C}_{i}: x=x_{i}(t)$ from $P$ downward, intersecting $x=x_{n}(t)$ at $B\left(t_{1}, x_{n}\left(t_{1}\right)\right)$. Then, we integrate (26) in the region $P A O B$ to get

$$
\begin{aligned}
\int_{\tilde{C}_{j}}\left(\lambda_{j}(u)-\lambda_{i}(u)\right)\left|w_{i}(t, x)\right| d t= & \int_{0}^{t_{0}}\left(\lambda_{1}(u)-\left(\lambda_{i}(u)\right)\left|\frac{\partial u_{i}\left(t, x_{1}(t)\right.}{\partial t}\right|\left|\frac{\partial t}{\partial x}\right| d t\right. \\
& +\int_{0}^{t_{1}}\left(\lambda_{n}(u)-\lambda_{i}(u)\right)\left|\frac{\partial u_{i}\left(t, x_{n}(t)\right)}{\partial t}\right|\left|\frac{\partial t}{\partial x}\right| d t
\end{aligned}
$$

Noting $i=1, \ldots, m i<j$, Equations (4), (13) and using the above procedure, we have

$$
\int_{\tilde{C}_{j}}\left|w_{i}(t, x)\right| d t \leq C\left(\int_{0}^{\infty}\left|\phi_{i}^{\prime}(t)\right| d t+\int_{0}^{\infty}\left|\psi_{i}^{\prime}(t)\right| d t\right) \leq C N_{2}
$$

If the point $A$ is on the characteristic boundary $x=x_{n}(t)$, using the same method as above cases we can get the desired estimates. Then, we can obtain 


$$
\tilde{W}_{1}(T)
$$

In the similar way, substituting $L_{j}$ for $\tilde{C}_{j}$ in the above cases we also can get

$$
\bar{W}_{1}(T) \leq C N_{2}
$$

In the following, we will get the estimate $W_{1}(T) \leq C N_{2}$. Integrating Equation (26) with respect to $x$ from $x_{1}(t)$ to $x_{n}(t)$ for any $t \in[0, T]$ leads to

$$
\begin{aligned}
& \int_{x_{1}(t)}^{x_{n}(t)} \partial_{t}\left|w_{i}(t, x)\right| d x=\lambda_{i}\left(u\left(t, x_{n}(t)\right)\right)\left|\frac{\partial u_{i}\left(t, x_{n}(t)\right)}{\partial x}\right|-\lambda_{i}\left(u\left(t, x_{1}(t)\right)\left|\frac{\partial u_{i}\left(t, x_{1}(t)\right)}{\partial x}\right|\right. \\
& \partial t \int_{x_{1}(t)}^{x_{n}(t)}\left|w_{i}(t, x)\right| d x=\left(\lambda_{n}-\lambda_{i}\right)(\psi(t))\left|\frac{\partial u_{i}\left(t, x_{n}(t)\right)}{\partial x}\right|-\left(\lambda_{1}-\lambda_{i}\right)(\psi(t))\left|\frac{\partial u_{i}\left(t, x_{1}(t)\right)}{\partial x}\right|
\end{aligned}
$$

Using the same procedure as (28), (29), we can get

$$
\int_{x_{1}(t)}^{x_{n}(t)}\left|w_{i}(t, x)\right| d x \leq C N_{2}
$$

Then, we can get the desired estimate.

Rewriting the Equation (25), we get

$$
\frac{\partial w_{i}}{\partial t}+\lambda_{i}(u) \frac{\partial w_{i}}{\partial x}=-\frac{\partial \lambda_{i}(u)}{\partial x} w_{i}
$$

Case 1 . When $i=1, \ldots, m$, we draw the $i$ th characteristic intersecting $x=x_{n}(t)$ with the point $\left(t_{0}, x_{n}\left(t_{0}\right)\right)$. Solving the ODE system we can get

$$
w_{i}\left(t, x_{i}(t)\right)=w_{i}\left(t_{0}, x_{n}\left(t_{0}\right)\right) e^{\int_{\bar{c}_{i}}-\frac{\partial \lambda_{i}(u)}{\partial x} d t}
$$

Noting $w_{i}\left(t_{0}, x_{n}\left(t_{0}\right)\right)=\frac{\psi_{i}^{\prime}\left(t_{0}\right)}{\lambda_{n}(u)}$ and the estimate (30), then

$$
\left|w_{i}\left(t, x_{i}(t)\right)\right| \leq C M e^{C \int_{\tilde{C}_{i}} \sum_{j \neq i}\left|w_{j}\right| d t} \leq C M e^{C \tilde{W}_{1}(T)} \leq C M e^{C N_{2}}
$$

Case 2. When $i=m+1, \ldots, n$ and the $i$ th characteristic intersecting $x=x_{1}(t)$ with a point $\left(t_{0}, x_{1}\left(t_{0}\right)\right)$, then

$$
w_{i}\left(t, x_{i}(t)\right)=w_{i}\left(t_{0}, x_{1}\left(t_{0}\right)\right) e^{\int_{\tilde{c}_{i}}-\frac{\partial \lambda_{i}(u)}{\lambda \mathrm{x}} d t}
$$

Then, we can get

$$
\left|w_{i}\left(t, x_{i}(t)\right)\right| \leq C M e^{C N_{2}}
$$

Then, we can obtain the estimate

$$
W_{\infty}(T) \leq C M e^{\mathrm{CN}_{2}}
$$


In the following we estimate $\tilde{U}_{1}(T)$.

Noting system (1), we denote the multiplier $H_{i} \in C^{1},(i=1, \ldots, n)$ such that,

$$
\frac{\partial\left(H_{i} u_{i}\right)}{\partial t}+\frac{\partial\left(\lambda_{i}(u) H_{i} u_{i}\right)}{\partial x}=0
$$

Noting (1), then

$$
\frac{\partial H_{i}}{\partial t}+\lambda_{i}(u) \frac{\partial H_{i}}{\partial x}=-\frac{\partial\left(\lambda_{i}(u)\right)}{\partial x} \cdot H_{i}
$$

Let $H_{i}\left(t, x_{1}(t)\right)=1$ and $H_{i}\left(t, x_{n}(t)\right)=1$ we can get

$$
H_{i}=e^{-\int \tilde{C}_{i} \frac{\partial \lambda_{i}(u(\tau, x))}{\partial x} d \tau}
$$

We note the system (1) is linearly degenerate, then

$$
\begin{aligned}
\left|\frac{\partial \lambda_{i}(u)}{\partial x}\right| & =\left|\sum_{j \neq i}^{n} \frac{\partial \lambda_{i}(u)}{\partial u_{j}} w_{j}\right| \leq C \sum_{j \neq i}\left|w_{j}\right| \\
e^{-C \sum_{j \neq i} \int_{\tilde{C}_{i}}\left|w_{j}\right| d t} & \leq H_{i} \leq e^{C \sum_{j \neq i} \tilde{C}_{\tilde{C}_{i}}\left|w_{j}\right| d t} \\
e^{-C \tilde{W}_{1}(T)} & \leq H_{i} \leq e^{C \tilde{W}_{1}(T)} \\
e^{-C N_{2}} & \leq H_{i} \leq e^{C N_{2}}
\end{aligned}
$$

Noting Equation (38), we can rewrite it as

$$
d\left(H_{i}\left|u_{i}\right|\left(d x-\lambda_{i}(u) d t\right)\right)=0
$$

There are only the following cases like we estimate $\tilde{W}_{1}(T)$ :

Case 1. We can integrate (40) in the region $A P B$ which is same to the case 1 of proof of $\tilde{W}_{1}(T)$ to get

$$
\int_{\tilde{C}_{j}} H_{i}\left|u_{i}\right|(t, x) d x \leq C \int_{t_{0}}^{t_{1}} H_{i}\left|u_{i}\right|\left(t, x_{1}(t)\right) d t
$$

Then

$$
\int_{\tilde{C}_{j}}\left|u_{i}\right|(t, x) d x \leq C e^{C N_{2}} \int_{0}^{+\infty}|\phi(t)| d t \leq C N_{1} e^{C N_{2}}
$$

Case 2. Integrating the Equation (40) in the region $P A O B$ which is same to the case 2 of proof of $\tilde{W}_{1}(T)$, we can get

$$
\begin{aligned}
\int_{c_{j}}\left(\lambda_{i}(u)-\lambda_{j}(u)\right) H_{i}\left|u_{i}\right|(t, x) d x= & \int_{0}^{t_{0}}\left(\lambda_{1}(u)-\lambda_{i}(u)\right) H_{i}\left|u_{i}\left(t, x_{1}(t)\right)\right| d t \\
& +\int_{0}^{t_{1}}\left(\lambda_{n}(u)-\lambda_{i}(u)\right) H_{i} \lambda_{i}(u)\left|u_{i}\left(t, x_{n}(t)\right)\right| d t
\end{aligned}
$$


Using the above procedures, we can get

$$
\int_{\tilde{C}_{j}}\left|u_{i}(t, x)\right| d x \leq C e^{C N_{2}}\left(\int_{0}^{+\infty}|\phi(t)| d t+\int_{0}^{+\infty}|\psi(t)| d t\right) \leq C N_{1} e^{C N_{2}}
$$

Then

$$
\tilde{U}_{1}(T) \leq C N_{1} e^{C N_{2}}
$$

In the similar way, substituting $L_{j}$ for $\tilde{C}_{j}$ we can get

$$
\begin{gathered}
\int_{L_{j}}\left|u_{i}(t, x)\right| d x \leq C N_{1} e^{C N_{2}} \\
\bar{U}_{1}(T) \leq C N_{1} e^{C N_{2}}
\end{gathered}
$$

Combining (24), (30), (31), (34), (37), (43), and (44) together we can obtain the conclusion of Lemma 2.1.

\section{Proof of Theorem 1.1.}

Noting the conclusion of Lemma 2.1, we can get

$$
\begin{aligned}
& \tilde{W}_{1}(\infty), \bar{W}_{1}(\infty), W_{1}(\infty) \leq C N_{2} \\
& \tilde{U}_{1}(\infty), \bar{U}_{1}(\infty), \leq C N_{1} e^{C N_{2}} \\
& W_{\infty}(\infty) \leq C M e^{C N_{2}} \\
& U_{\infty}(\infty) \leq C
\end{aligned}
$$

Therefore, we can obtain that the system (1), (5), and (6) have global classical solutions on the domain $D=\left\{(t, x) \mid t \geq 0, x_{1}(t) \leq x \leq x_{n}(t)\right\}$.

\section{Asymptotic behavior of global classical solutions}

In this section, under the assumption of the leftmost and rightmost characteristics, we will study the asymptotic behavior of the global classical solutions of system (1), (5), and (6) and give the proof of Theorem 1.2.

Let

$$
\frac{D}{D_{i} t}=\frac{\partial}{\partial t}+\lambda_{i}(0) \frac{\partial}{\partial x}
$$

Obviously,

$$
\frac{D}{D_{i} t}=\frac{d}{d_{i} t}+\left(\lambda_{i}(0)-\lambda_{i}(u)\right) \frac{\partial}{\partial x}
$$

where $\frac{d}{d_{i} t}=\frac{\partial}{\partial t}+\lambda_{i}(u) \frac{\partial}{\partial x}$. Thus, noting system (1)

$$
\frac{D u_{i}}{D_{i} t}=\left(\lambda_{i}(0)-\lambda_{i}(u)\right) \frac{\partial u_{i}}{\partial x}
$$


Using the Hadamard's Lemma, we can obtain

$$
\frac{D u_{i}}{D_{i} t}=-\sum_{j \neq i}\left\{\Lambda_{i j}(u) u_{j} w_{i}\right\}
$$

where $\Lambda_{i j}(u)=\int_{0}^{1} \frac{\partial \lambda_{i}\left(s u_{1}, \ldots, s u_{i}-1, u_{i}, s u_{i}+1, \ldots, s u_{n}\right)}{\partial u_{j}} d s$.

For any fixed point $(t, x) \in D$, Passing through $(t, x)$, we draw down the characteristic $x=x_{r}(t)$, which intersect with the characteristic boundary in the point $\left(x_{r}^{-1}(\alpha), \alpha\right)$. Then $\alpha=x-\lambda_{i}(0)\left(t-x_{r}^{-1}(\alpha)\right.$ ) (where $r=1$, when $i=m+1, \ldots, n$ or $r=n$, when $i=$ $1, \ldots, m)$.

Then, it follows from Equation (51) that

$$
\begin{aligned}
u_{i}(t, x) & =u_{i}\left(t, \alpha+\lambda_{i}(0)\left(t-x_{r}^{-1}(\alpha)\right)\right) \\
& =u_{i}\left(x_{r}^{-1}(\alpha), \alpha\right)-\int_{x_{r}^{-1}(\alpha)}^{t} \sum_{j \neq i}\left\{\Lambda_{i j}(u) u_{j} w_{i}\left(s, \alpha+\lambda_{i}(0)\left(s-x_{r}^{-1}(\alpha)\right)\right)\right\} d s
\end{aligned}
$$

Noting (46) and (47), we can get

$$
\left|\int_{k_{r}^{-1}(\alpha)}^{t} \sum_{j \neq i}\left\{\Lambda_{i j}(u) u_{j} w_{i}\right\} d s\right| \leq C W_{\infty}(\infty) \bar{U}_{1}(\infty) \leq C M N_{1} e^{C N_{2}}
$$

This implies that the integral $\int_{x_{r}^{-1}(\alpha)}^{t} \sum_{j \neq i}\left\{\Lambda_{i j}(u) u_{j} w_{i}\left(s, \alpha+\lambda_{i}(0)\left(s-x_{r}^{-1}(\alpha)\right)\right)\right\} d s$ converges uniformly for $\alpha \in R$. Then, there exists a unique function $\Phi_{i}(\alpha)$ such that,

$$
u_{i}(t, x) \rightarrow \Phi_{i}(\alpha), \quad t \rightarrow+\infty
$$

Moreover, noting (13) and Equations (52), (53), we have

$$
\left|\Phi_{i}(\alpha)\right| \leq C\left(1+M N_{1}\right) e^{C N_{2}}
$$

In what follows, we will study the regularity of $\Phi(\alpha)$. Noting Equation (52), we can get $\Phi(\alpha) \in C^{0}(R)$. From any fixed point $A(t, x)=\left(t, a+\lambda_{i}(0)\left(t-x_{r}^{-1}(\alpha)\right)\right)$, we draw a characteristic $\tilde{C}_{j}$ intersecting the boundary $x=x_{1}(t)$ or $x=x_{n}(t)$ at $\left(x_{r}^{-1}\left(\theta_{i}(t, \alpha)\right), \theta_{i}(t, \alpha)\right)$.

Then, integrating it along the $i$ th characteristic, we obtain

$$
\alpha+\lambda_{i}(0)\left(t-x_{r}^{-1}(\alpha)\right)=\theta(t, \alpha)+\int_{x_{r}^{-1}\left(\theta_{i}(t, \alpha)\right)}^{t} \lambda_{i}\left(u\left(\tau, x_{i}\left(\tau, \theta_{i}(t, \alpha)\right)\right)\right) d \tau
$$

Then, we can get the following Lemma

Lemma 3.1 Under the assumptions of Theorem 1.1, for the $\theta_{i}(t, \alpha)$ defined above, there exists a unique $\vartheta_{i}(\alpha)$, such that

$$
\lim _{t \rightarrow+\infty} \theta_{i}(t, \alpha)=\vartheta_{i}(\alpha)
$$


Proof. Using the Hardarmad's formula, we can rewrite (55) as following

$$
\theta_{i}(t, \alpha)=\alpha+\int_{x_{r}^{-1}\left(\theta_{i}(t, \alpha)\right)}^{t} \Lambda_{i j} u_{j}\left(\tau, x_{i}\left(\tau, \theta_{i}(t, \alpha)\right)\right) d \tau+\lambda_{i}(0)\left(x_{r}^{-1}\left(\theta_{i}(t, \alpha)\right)-x_{r}^{-1}(\alpha)\right)
$$

where

$$
\Lambda_{i j}=-\int_{0}^{1} \frac{\partial \lambda_{i}\left(s u_{1}, \ldots, s u_{i-1}, u_{i}, s u_{i+1}, \ldots, s u_{n}\right)}{\partial u_{j}} d s .
$$

are $C^{1}$ functions. Therefore

$$
\theta_{i}(t, \alpha)-\lambda_{i}(0) x_{r}^{-1}\left(\theta_{i}(t, \alpha)\right)=\alpha+\int_{x_{r}^{-1}\left(\theta_{i}(t, \alpha)\right)}^{t} \Lambda_{i j} u_{j}\left(\tau, x_{i}\left(\tau, \theta_{i}(t, \alpha)\right)\right) d \tau-\lambda_{i}(0) x_{r}^{-1}(\alpha)
$$

where $r$ is either 1 or $n$. Then, we know that when $t$ tends to $\infty$, the right hand of (58) convergence absolutely. For any given $\alpha$, the right hand of (57) convergence to some function with respect to $\alpha$. That implies that there exists a unique function $\vartheta(\alpha)$, such that

$$
\lim _{t \rightarrow+\infty} \theta_{i}(t, \alpha)=\vartheta_{i}(\alpha)
$$

Lemma 3.2 Under the assumptions of Theorem 1.1, for any given point $\left(x_{r}^{-1}(\alpha), \alpha\right)$ on the boundary, there exists a unique function $\Psi_{i}(\vartheta(\alpha)) \in C^{0}$, such that

$$
\lim _{t \rightarrow+\infty} w_{i}\left(t, \alpha+\lambda_{i}(0)\left(t-x_{r}^{-1}(\alpha)\right)\right)=\Psi_{i}\left(\vartheta_{i}(\alpha)\right) .
$$

uniformly for any $\alpha L R$.

Proof. Noting

$$
w_{i}\left(t, \alpha+\lambda_{i}(0)\left(t-x_{r}^{-1}(\alpha)\right)\right)=w_{i}\left(t, x_{i}\left(t, \theta_{i}(t, \alpha)\right)\right) .
$$

In the following, we prove that there exists a unique $\Psi_{i}(\vartheta(\alpha)) \in C^{0}$, such that

$$
\left.\lim _{t \rightarrow+\infty} w_{i}\left(t, x_{i}\left(t, \theta_{i}(t, \alpha)\right)\right)\right)=\Psi_{i}\left(>\vartheta_{i}(\alpha)\right) .
$$

Integrating (35) along the $i$ th characteristic $\tilde{C}_{j}$ gives

$$
\begin{aligned}
\left.w_{i}\left(t, x_{i}(t, \alpha)\right)\right) & =w_{i}\left(x_{r}^{-1}\left(\theta_{i}(t, \alpha)\right), \theta_{i}(t, \alpha)\right) \\
& +\int_{x_{r}^{-1}\left(\theta_{i}(t, \alpha)\right)}^{t} \sum_{j \neq i} \gamma_{i j}(u) w_{j} w_{i}\left(\tau, x_{i}\left(\tau, \theta_{i}(t, \alpha)\right)\right) d \tau
\end{aligned}
$$

where $\gamma_{i j}(u)=\frac{\partial \lambda_{i}(u)}{\partial u_{j}}$. Noting (45) and (47), we can get

$$
\left|\int_{x_{r}^{-1}\left(\theta_{i}(t, \alpha)\right)}^{t} \sum_{j \neq i} \gamma_{i j}(u) w_{j} w_{i}\left(\tau, x_{i}\left(\tau, \theta_{i}(t, \alpha)\right)\right) d \tau\right| \leq C M N_{2} e^{C N_{2}}
$$

Then, as $t$ tends to $+\infty$, the integrals in the right-hand side of (63) convergence, i.e., there exists a unique function $\Psi_{i}\left(\vartheta_{i}(\alpha)\right)$, such that 


$$
\left.\lim _{t \rightarrow \infty} w_{i}\left(t, x_{i}(t, \alpha)\right)\right)=\Psi_{i}\left(\vartheta_{i}(\alpha)\right)
$$

\section{Lemma 3.3}

$$
\begin{aligned}
& \frac{d \Phi_{i}(\alpha)}{d \alpha}=\left(1-\frac{\lambda_{i}(0)}{\lambda_{i}\left(x_{1}^{-1}(\alpha), \alpha\right)}\right) \Psi_{i}\left(\vartheta_{i}(\alpha)\right) \\
& \frac{d \Phi_{i}(\alpha)}{d \alpha}=\left(1-\frac{\lambda_{i}(0)}{\lambda_{n}\left(x_{n}^{-1}(\alpha), \alpha\right)}\right) \Psi_{i}\left(\vartheta_{i}(\alpha)\right)
\end{aligned}
$$

Proof. For any fixed $\alpha \in R$, we calculate

$$
\begin{aligned}
\frac{d \Phi_{i}(\alpha)}{d \alpha}= & \lim _{\Delta \alpha \rightarrow \infty} \frac{\Phi_{i}(\alpha+\Delta \alpha)-\Phi_{i}(\alpha)}{\Delta \alpha} \\
= & \lim _{\Delta \alpha \rightarrow 0} \lim _{t \rightarrow+\infty} \frac{u_{i}\left(t, \alpha+\Delta \alpha+\lambda_{i}(0)\left(t-x_{r}^{-1}(\alpha+\Delta \alpha)\right)\right)-u_{i}\left(t, \alpha+\lambda_{i}(0)\left(t-x_{r}^{-1}(\alpha)\right)\right)}{\Delta \alpha} \\
= & \lim _{t \rightarrow+\infty \Delta \alpha \rightarrow 0} \lim _{\Delta \alpha \rightarrow 0} \frac{u_{i}\left(t, \alpha+\Delta \alpha+\lambda_{i}(0)\left(t-x_{r}^{-1}(\alpha+\Delta \alpha)\right)\right)-u_{i}\left(t, \alpha+\lambda_{i}(0)\left(t-x_{r}^{-1}(\alpha)\right)\right)}{\Delta \alpha} \\
= & \lim _{t \rightarrow+\infty} \lim _{\Delta \alpha \rightarrow 0}\left[\frac{u_{i}\left(t, \alpha+\lambda_{i}(0)\left(t-x_{r}^{-1}(\alpha)\right)+\Delta \alpha-\lambda_{i}(0) \frac{x_{r}^{-1}(\alpha+\Delta \alpha)-x_{r}^{-1}(x)}{\Delta \alpha} \cdot \Delta \alpha\right)}{\Delta \alpha}\right. \\
& \left.-\frac{u_{i}\left(t, \alpha+\lambda_{i}(0)\left(t-x_{r}^{-1}(\alpha)\right)\right)}{\Delta \alpha}\right]
\end{aligned}
$$

Thus, when $i=1, \ldots, s$, i.e., $r=1$, we can get

$$
\begin{aligned}
\frac{d \Phi_{i}(\alpha)}{d \alpha} & =\left(1-\frac{\lambda_{i}(0)}{\lambda_{1}\left(x_{1}^{-1}(\alpha), \alpha\right)}\right) \lim _{t \rightarrow+\infty} w_{i}\left(t, \alpha+\lambda_{i}(0)\left(t-x_{1}^{-1}(\alpha)\right)\right) \\
& =\left(1-\frac{\lambda_{i}(0)}{\lambda_{1}\left(x_{1}^{-1}(\alpha), \alpha\right)}\right) \Psi_{i}\left(\vartheta_{i}(\alpha)\right) .
\end{aligned}
$$

Using the similar procedure, when $\alpha>0$, i.e., $i=s+1, \ldots, n$ and $r=n$, we can get

$$
\frac{d \Phi_{i}(\alpha)}{d \alpha}=\left(1-\frac{\lambda_{i}(0)}{\lambda_{n}\left(x_{n}^{-1}(\alpha), \alpha\right)}\right) \Psi_{i}\left(\vartheta_{i}(\alpha)\right) .
$$

Noting the above lemmas, we get the conclusion of Theorem 1.2.

\section{Applications}

Recent years observations of the luminosity of type Ia distant supernovae point towards an accelerated expansion of the universe, which implies that the pressure $p$ and the energy density $\rho$ of the universe should violate the strong energy condition, i.e., $\rho+3 p$ $<0$. Here, we consider a recently proposed class of simple cosmological models based on the use of peculiar perfect fluids [19]. In the simplest case, we study the model of a universe filled with the so called Chaplygin gas, which is a perfect fluid characterized by the following equation of state $p=-\frac{A}{\rho}=-A \tau$, where $A$ is a positive constant.

In Lagrange coordinate, the $1 \mathrm{D}$ gas dynamics equations in isentropic case can be written as 


$$
\left\{\begin{array}{l}
\tau_{t}-u_{x}=0 \\
u_{t}+p(\tau)_{x}=0
\end{array}\right.
$$

Noting (69), in isentropic case we can get the system of one dimensional Chaplygin gas model

$$
\left\{\begin{array}{l}
\tau_{t}-u_{x}=0 \\
u_{t}-A \tau_{x}=0
\end{array}\right.
$$

Nothing systems (70) is linear systems, it is easy to get the eigenvalues

$$
\lambda_{+}=\sqrt{A_{1}} \quad \lambda_{-}=-\sqrt{A} .
$$

and left eigenvectors

$$
l_{+}=(\sqrt{A}, 1), l_{-}=(-\sqrt{A}, 1)
$$

Introduce the Riemann invariants

$$
v_{1}=u+\sqrt{A} \tau, \quad v_{2}=u-\sqrt{A} \tau
$$

we can rewrite the system as following

$$
\left\{\begin{array}{l}
\frac{\partial v_{1}}{\partial t}-\sqrt{A} \frac{\partial v_{1}}{\partial x}=0 \\
\frac{\partial v_{2}}{\partial t}-\sqrt{A} \frac{\partial v_{2}}{\partial x}=0
\end{array}\right.
$$

Consider the Goursat problem for system (69) with following characteristic boundary conditions:

$$
x=-\sqrt{A} t: \tau=\tau_{-}(t), u=u_{-}(t) ; \quad x=\sqrt{A} t: \tau=\tau_{+}(t), u=u_{+}(t)
$$

Then, the above system satisfies the assumptions of Theorems 1.1 and 1.2. More precisely, we can get the following theorems:

Theorem 4.1. The Goursat problem (69) and (75) admits a unique global $C^{1}$ solution $(\tau, \mathrm{u})(t, x)$ on the domain $D=\{(t, x) \mid t \geq 0,-\sqrt{A} t \leq x \leq \sqrt{A} t\}$.

Theorem 4.2. There exists a unique piece-wised $C^{1}$ vector-value function $\Phi(x)=\left(\Phi_{1}\right.$ $\left.(x), \ldots, \Phi_{n}(x)\right)^{T}$ such that

$$
(\tau, u)(t, x) \rightarrow \sum_{i=1}^{n} \Phi_{i}(x-\sqrt{A} t) e_{i}, \quad t \rightarrow+\infty
$$

uniformly as $t$ tends to infinity, where $e_{i}=\left(0, \ldots, 0,1^{i}, 0, \ldots, 0\right)^{T}$.

\section{Acknowledgements}

The first author was supported by NSFC-Tianyuan Special Foundation (No. 11126058), Excellent Young Teachers Program of Shanghai and the Shanghai Leading Academic Discipline Project (No. J50101). The second author was supported by China Postdoctoral Science Foundation (No. 2011M501295) and the Fundamental Research Funds for the Central Universities (No. 2011QNZT102). The authors would like to thank Professor Zhou Yi for his guidance and encouragements. 


\section{Author details}

${ }^{1}$ Department of Mathematics, Shanghai University, Shanghai 200444, China ${ }^{2}$ Key Laboratory of Metallogenic Prediction of Nonferrous Metals, Ministry of Education, School of Geosciences and Info-Physics, Central South University, Changsha 410083, China ${ }^{3}$ School of Mathematics and Statistics, Central South University, Changsha 410075, China

\section{Authors' contributions}

The authors declare that the study was realized in collaboration with the same responsibility. All authors read and approved the final manuscript.

\section{Competing interests}

The authors declare that they have no competing interests.

Received: 27 October 2011 Accepted: 3 April 2012 Published: 3 April 2012

\section{References}

1. Bressan, A: Contractive metrics for nonlinear hyperbolic system. Nat Genet. 37, 409- (1988)

2. Li, TT: Global classical solutions for qusilinear hyperbolic systems. In Research in Appl Math, vol. 32,Wiley/Masson, New York (1994)

3. $L i, \Pi$, Zhou, $Y$, Kong, DX: Weak linear degeneracy and global classical solutions for general qusilinear hyperbolic systems. Comm Partial Diff Equ. 19, 1263-1317 (1994)

4. Li, $\Pi$, Zhou, Y, Kong, DX: Global classical solutions for general qusilinear hyperbolic systems with decay initial data. Nonl Anal. 28, 1299-1322 (1997)

5. $L i, \Pi$, Peng, $Y$ J: Global $C^{1}$ solution to the initial-boundary value problem for dioagal hyperbolic systems with linearly degenerate characteristics. J Partial Diff Equ. 16, 8-17 (2003)

6. Li, TT, Peng, YJ: The mixed initial-boundary value problem for reducible qusilinear hyperbolic systems with linearly degenerate characteristics. Nonlinear Anal. 52, 573-583 (2003)

7. Zhou, Y: Global classical solutions to qusilinear hyperbolic systems with weak linear degeneracy. Chin Ann Math Ser B. 25, 37-56 (2004)

8. Kong, DX, Yang, T: Asymptotic behavior of global classical solutions of qusilinear hy-perbolic systems. Comm Part Diff Equ. 28, 1203-1220 (2003)

9. Dai, WR, Kong, DX: Asymptotic behavior of global classical solutions of general qusilinear hyperbolic systems with weakly linearly degeneracy. Chinese Ann Math Ser B. 27(3):263-286 (2006)

10. Dai, WR, Kong, DX: Global existence and asymptotic behavior of classical solutions of qusilinear hyperbolic systems with linearly degenerate characteristic fields. J Diff Equ. 251(3):127-165 (2007)

11. Liu, JL, Zhou, Y: Asymptotic behavior of global classical solutions of diagonalizable qusilinear hyperbolic systems. Math Meth Appl Sci. 30, 479-500 (2007)

12. Li, TT, Wang, LB: Global classical solutions to a kind of mixed initial-boundary value problem for qusilinear hyperbolic systems. Discrete Cont Dyn Syst. 12, 59-78 (2005)

13. Zhang, JG: Asymptotic behavior of global classical solutions to a kind of mixed initial-boundary value problem for quasilinear hyperbolic systems. J Partial Diff Equ. 20, 114-130 (2007)

14. Liu, J: The mixed initial-boundary value problem for diagonalizable quasilinear hyperbolic systems in the first quadrant. Nonlinear Anal. 72(1):533-545 (2010)

15. Zhou, Y: The Goursat problem for reducible quasilinear hyperbolic systems. Chinese Ann Math Ser A. 13, 437-441 (1992)

16. Liu, CM, Liu, JL: Goursat problem for the first order quasilinear hyperbolic systems (Sumitted to Acta Mathematica Sinica, English Series).

17. Liu, JL, Pan, KJ: Asymptotic behavior of global classical solutions to Goursat problem of quasilinear hyperbolic systems. (Accepted by J Math Anal Appl).

18. Duan, YZ: Asymptotic behavior of global classical solutions of reducible qusilinear hy-perbolic systems with characteristic boundaries. J Math Anal Appl. 351, 186-205 (2009)

19. Gorini, V, Kamenshchik, A, Moschella, U, Pasquier, V: Can the Chaplygin gas be a plausible model for dark energy? Phys. Rev D. 67, 063509 (2003)

doi:10.1186/1687-2770-2012-36

Cite this article as: Liu and Pan: Global existence and asymptotic behavior of classical solutions to Goursat

problem for diagonalizable quasilinear hyperbolic system. Boundary Value Problems 2012 2012:36. 